\title{
ISSUES IN MEDICINE The spread of carbapenem-resistant Enterobacteriaceae in South Africa: Risk factors for acquisition and prevention
}

\author{
Adrian Brink, Jennifer Coetzee, Cornelis Clay, Craig Corcoran, Johan van Greune, J D Deetlefs, Louise Nutt, Charles Feldman, Guy \\ Richards, Patrice Nordmann, Laurent Poirel
}

New, effective antibiotics are only likely to become available in 15 - 20 years. To prevent deaths from untreatable Gram-negative infections in South Africa, the rights of any doctor, whether in general or in hospital practice, to indiscriminately prescribe whatever antibiotic they wish, and in whatever fashion, must be challenged. Furthermore, although prevention of the emergence and subsequent spread of carbapenem-resistant Enterobacteriaceae
(CRE) has focused on acute and chronic care facilities and inter alia on antibiotic exposure in these institutions, CRE may soon become an issue within entire communities, highlighting a role for public health authorities in CRE prevention efforts.

S Afr Med J 2012;102(7):599-601.

\section{Background}

In 2008, we stated that 'the die was cast' regarding the emergence and nationwide spread of extensively resistant (XDR) and pandrug-resistant (PDR) Gram-negative fermentative bacteria such as Escherichia coli and Klebsiella pneumoniae. ${ }^{1}$ This prediction was based on the fact that suboptimal antibiotic management as a whole (excessive duration, use of multiple often inappropriate or unnecessary agents, and a virtual absence of de-escalation) was rife in clinical practice in most institutions, and that prescribers ignored antibiotic 'stewardship' as a means of combating the emergence of XDR and PDR Gram-negative bacilli (GNB).

Subsequently, carbapenem-resistant Enterobacteriaceae (CRE) have indeed become our 'worst nightmare', locally and internationally, and pose a major threat to the viability of all currently available antibiotics. ${ }^{2-4}$ These GNB are resistant to all standard antimicrobial

Adrian Brink is a clinical microbiologist in the Department of Clinical Microbiology, Ampath National Laboratory Services, Milpark Hospital, Johannesburg.

Jennifer Coetzee is a clinical microbiologist, Cornelis Clay a molecular scientist and Craig Corcoran a clinical virologist in the Department of Clinical Microbiology and Molecular Biology, Ampath National Laboratory Services, National Reference Laboratory, Centurion, Gauteng.

Johan van Greune and J D Deetlefs are clinical pathologists at Ampath National Laboratory Services, Cape Town, and Louise Nutt is a clinical microbiologist at Ampath National Laboratory Services, Port Elizabeth.

Guy Richards (intensivist) and Charles Feldman (pulmonologist) are in the Department of Critical Care and Division of Pulmonology, Charlotte Maxeke Johannesburg Academic Hospital, and University of the Witwatersrand, Johannesburg.

Patrice Nordmann and Laurent Poirel (microbiologists) are affiliated to the Service de Bacteriologie-Virologie, Hospital de Bicetre, Assistance Publique/Hopitaux de Paris, Faculte de Medecine et Universite Paris-Sud, K-Bicetre, France. agents and salvage therapy with tigecycline, colistin and/or fosfomycin has become the last resort in life-threatening infections. Numerous outbreaks and epidemics with these organisms have been reported, they have become endemic in several institutions, and increased mortality has been ascribed to them. ${ }^{2-4}$ The imminent threat of untreatable infections is highlighted by the recent emergence, and inter-hospital spread in Greece, of CRE resistant even to colistin. ${ }^{5,6}$

\section{Carbapenemases}

Carbapenem resistance among Enterobacteriaceae can be conferred by several genetic mechanisms, but epidemiologically the most important of them results in the production of beta-lactamases (carbapenemases), which hydrolyse carbapenems and most other beta-lactams. These genes mostly reside on large plasmids which frequently contain other resistance determinants, such as those that confer resistance to the aminoglycosides and reduced susceptibility to the fluoroquinolones.

The carbapenemases belong to different classes and include:

- K. pneumoniae carbapenemases (KPCs) and Guiana extendedspectrum $\beta$-lactamases (GESs) (class A)

- the metallo- $\beta$-lactamases (MBLs), such as the Verona integronencoded MBLs (VIMs) and the recently described New Delhi metallo- $\beta$-lactamases (NDM-1) (class B)

- oxacillinase-type carbapenemases such as OXA-48 and its derivates, which also occur in Enterobacteriaceae (class D).

Since 1 March 2010 the Molecular Biology Laboratory at the Ampath National Reference Laboratory has been screening Enterobacteriaceae for the presence of these novel genes, and recently the emergence of NDM- 1 for the first time in South Africa, and KPC-2 for the first time in Africa, was documented among clinical isolates of $K$. pneumoniae and Enterobacter cloacae in hospitalised patients in Johannesburg and Pretoria, respectively. ${ }^{4}$ As depicted in Fig. 1, the emergence of the broad-spectrum antibiotic-inactivating enzyme, OXA-48, and its derivatives among Enterobacteriaceae from hospitalised patients in Johannesburg, Cape Town and Port Elizabeth has been confirmed (unpublished, J Coetzee, February 2012). Furthermore, VIMs have been detected in K. pneumoniae in Johannesburg and GESs in Enterobacteriaceae in hospitals in Cape Town $(K$. pneumoniae), Bloemfontein (K. oxytoca), Witbank (E. cloacae) and Port Elizabeth (Serratia marcescens) (unpublished, J Coetzee, February 2012).

(a) 


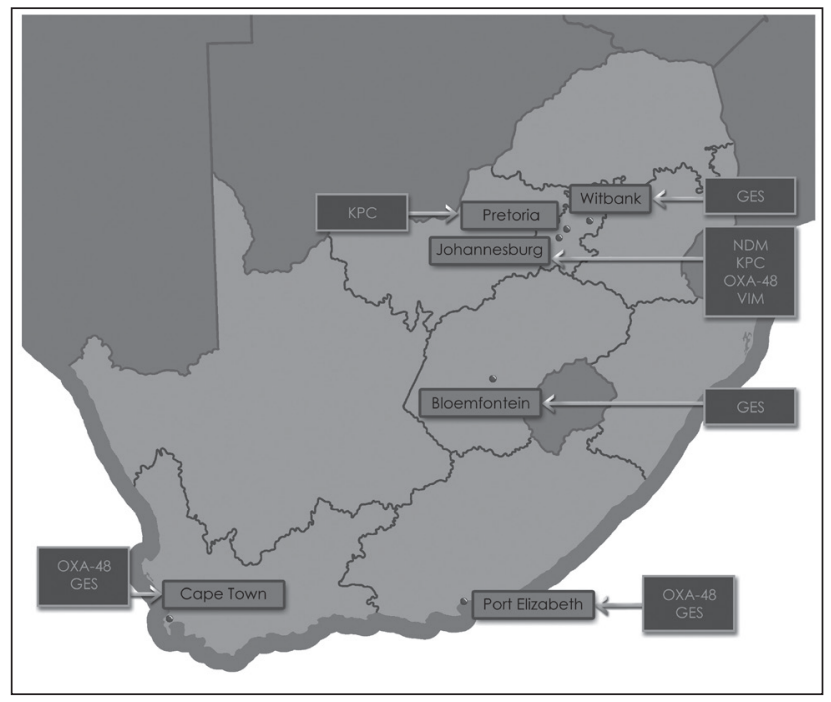

Fig. 1. Emergence and spread of carbapenemase genes among Enterobacteriaceae in South Africa (KPC $=$ Klebsiella pneumoniae carbapenemases; GES = Guiana extended-spectrum $\beta$-lactamases; $N D M=$ New Delhi metallo- $\beta$-lactamases $;$ OXA-48 = oxacillinase-type carbapenemases; VIM = Verona integron-encoded metallo- $\beta$-lactamases).

\section{Risk factors for acquisition Antibiotic exposure}

Exposure to antibiotics is a prominent risk factor for CRE. Specific antibiotics and specific antibiotic classes have frequently been implicated as risk factors for colonisation or infection with CRE, and these include all the carbapenems, cephalosporins, fluoroquinolones, aminoglycosides and $\beta$-lactam/ $\beta$-lactamase inhibitors. ${ }^{3}$ Studies have also shown that prior carbapenem therapy is not a prerequisite for carbapenem resistance among E. coli or K. pneumoniae., The plasmids that confer such resistance frequently carry additional resistance determinants that confer cross-resistance to most other antibiotic classes. Consequently, prior use of any antibiotic may select for a carbapenemase-producing GNB.

\section{Healthcare exposure}

The major risk factors for acquiring KPCs (colonisation and/or infection) are similar to those for extended-spectrum $\beta$-lactamases (ESBLs), the prevalence of which is extremely high in South Africa. For example, the ESBL rate for K. pneumoniae cultured from complicated intra-abdominal infections in private hospitals is $41.2 \%$ and that for bacteraemic isolates in the public sector $55-74 \%{ }^{7,8}$

These risk factors include: ${ }^{3,9}$

- organ or stem cell transplantation

- intensive care unit admission

- poor functional status

- severe illness

- mechanical ventilation

- prolonged hospitalisation

- surgery.

Similar to ESBLs, it also appears that long-term care facilities are reservoirs of KPCs because they act as a point of convergence of patients at high risk, amplified by cross-transmission, and this facilitates regional dissemination. ${ }^{3,10}$

Emerging evidence suggests that the presence of a high 'invasivedevice score' is also a predictor of CRE. For K. pneumoniae, the presence of a central venous line (odds ratio (OR) 5.22; 95\% confidence interval (CI) $2.38-11.46 ; p<0.001)$ and a urinary catheter
(OR 7.53; 95\% CI 3.49 - 16.26; $p<0.001$ ) are significantly associated with acquisition of KPC. ${ }^{9}$

Transfer of patients who have been hospitalised in medical facilities or countries where endemicity of CRE isolates has been established is also a major risk factor.

\section{Prevention of CRE}

Rapid routine molecular detection of CRE is essential to optimise therapy and improve outcomes, and to limit the spread of such resistance through aggressive infection control, including the screening of high-risk patients and the facilitation of 'search and contain' tactics. ${ }^{4}$ Detection of carriers of CRE is currently by far the most important factor preventing further dissemination in hospital settings.

\section{Antibiotic utilisation}

Delaying the emergence of carbapenem resistance, particularly in areas of South Africa where resistance is still uncommon, can decrease the impact of CRE. In this regard, antibiotic stewardship programmes (ASPs) are crucial to prevent the emergence of CRE. Because so few agents are available to treat the present population of XDR organisms, it is extremely difficult to study restrictive formulary interventions, and if they were employed they would be unlikely to be successful. Rather than targeting a specific class of antibiotics or limiting use of specific agents, an overall reduction in antibiotic burden is recommended as a focus for ASPs. ${ }^{3}$

Cumulative antibiotic exposure is likely to be the most important factor determining risk for developing a CRE infection. In a 4-year case-control study $(N=102)$, the only covariate independently associated with CRE in multivariate analysis was the cumulative number of prior antibiotic exposures: ORs (95\% CI) were 1.43 (1.19 - 1.72), 2.05 (1.70 - 2.47) and 2.93 $(2.43-3.53)$ for 1,2 and $\geq 3$ antibiotic exposures, respectively. ${ }^{11}$

It also appears that not only is prior cumulative antibiotic exposure a risk factor, but that the risk increases with increasing duration of treatment. Kritsotakis et al. demonstrated that this was the case for $\beta$-lactam/ $\beta$-lactamase inhibitor combinations (OR 1.15 increase in resistance per day of use; $p=0.001$ ) and also for the fluoroquinolones, where increased duration of treatment amplified the effect of exposure to carbapenems (OR 1.02 for interaction term, $p=0.0009$ ). ${ }^{9}$

Suboptimal dosing may also be a contributing factor for the development of resistance, and as a consequence it is advisable that administration of those agents that are still left for treatment, such as colistin, should be optimised in terms of dose and by avoiding use of these agents on their own. ${ }^{12-14}$

Recognising the extent to which antibiotics are inappropriately used in South Africa, the Federation of Infectious Diseases of Southern Africa (FIDSSA) recently hosted the 1st South African Antibiotic Stewardship Program (SAASP) in Johannesburg. While ASPs were initiated at pilot hospitals in South Africa, outpatient antibiotic use in this country has not been addressed and it is now crucial also to target general practitioners and the South African public as a whole. However, experience elsewhere suggests that it is not possible to influence antibiotic prescribers without instituting restrictive or even what may be perceived as punitive measures. How this will be implemented in South Africa remains unclear.

\section{Management of invasive devices}

CRE have been identified from device-associated infections, particularly catheter-associated urinary tract infections. Limiting the use of invasive devices and using defined strategies that prevent their contamination and infection might be another important intervention to prevent occurrence and spread of CRE. ${ }^{3}$ These strategies should be urgently implemented in all our hospitals. 
Urinary catheters should only be used in those patients who have appropriate indications, should be removed as soon as possible, and should have sterile, closed drainage systems. Aseptic insertion and maintenance of all catheters, according to protocols laid out in catheter 'bundles', are essential and constitute best care.

The Best Care ... Always! (BCA) campaign was initiated in 2009 to support southern African healthcare organisations in implementing specific, internationally recognised, evidence-based interventions that enhance patient safety and constitute current best practice in hospital care. ${ }^{15}$ Early BCA results are encouraging and significant strides have been made since some of these bundles have been implemented in over 200 South African hospitals. For example, in 40 and 42 acute-care hospitals of two private hospital groups, a significant decrease in central line infections from 5.24 and 5.78 per 1000 catheter days to $1.34(p<0.0001)$ and $1.27(p<0.0001)$ line days, respectively, was recently confirmed over an 18 -month period (unpublished, A J Brink, February 2012).

Conflict of interests declaration. No external funding was received for the molecular work at the Ampath Molecular Laboratory. $\mathrm{AB}$ has received recent research funding from Merck and Sanofi-Aventis, and has served on the advisory board of MSD, Aspen and Pfizer and on speakers' bureau for GlaxoSmithKline (GSK), Merck, Pfizer and Sanofi-Aventis. JvG received honoraria for lectures or assistance for congress travel from the following companies in relation to antibiotics they manufacture or market: AstraZeneca, Pfizer, MSD and Aspen. GAR has served on the speaker's bureau of and/or received funding for congress travel from Sanofi-Aventis, Pfizer, Merck and Co. and Bristol-Myers Squibb, Astra-Zeneca, Roche, Winthrop, Aspen, Bayer, GSK, Janssen, Fresenius Kabi and Abbott. CF has acted on the advisory board or received honoraria for lectures or assistance for congress travel from the following companies in relation to antibiotics they manufacture or market: Abbott, Merck, Aspen-GSK, Pfizer, Cipla, Astra-Zeneca and Sanofi-Aventis Other authors: no disclosure.

\section{References}

1. Brink AJ, Feldman C, Richards GA, et al. Emergence of extensive drug resistance (XDR) among Gramnegative bacilli in South Africa looms nearer. S Afr Med J 2008;98:586-592.

2. Nordmann P, Naas T, Poirel L. Global spread of carbapenemase-producing Enterobacteriaceae. Emer Infect Dis 2011;17:1791-1798. [http://doi.org/10.3201/eid1710.110655]

3. Gupta N, Lumbago BM, Patel JB, et al. Carbapenem-resistant Enterobacteriaceae: Epidemiology and prevention. Clin Infect Dis 2011;53:60-67. [http://doi.org/10.1093/cid/cir202]

4. Brink AJ, Coetzee J, Clay C, et al. Emergence of New Delhi metallo-beta-lactamase (NDM-1) and Klebsiell pneumonise car pneumoniae carbapenemase
org/10.1128/JCM.05956-11]

5. Kontopoulou K, Protonotariou E, Vasilakos K, et al. Hospital outbreak caused by Klebsiella pneumoniae producing KPC-2 $\beta$-lactamase resistant to colistin. J Hosp Infect 2010;76:70-73. [http://doi.org/10.1016/j.jhin.2010.03.021] Zarkotou O, Pournaras S, Voulgari E, et al. Risk factors and outcomes associated with acquisition of 6. Zarkotou O, Pournaras S, Voulgari E, et al. Risk factors and outcomes associated with acquisition of
colistin-resistant KPC-producing Klebsiella pneumoniae: a matched case-control study. J Clin Microbiol colistin-resistant KPC-producing Klebsiella pneumoniae: a

2010;48:2271-2274. [http://doi.org/10.1128//CM.02301-09]
Brink AJ, Botha RF, Poswa X, et al. Antimicrobial susceptibility of Gram-negative pathogens isolated from patients with complicated intra-abdominal infections in South African hospitals (SMART Study 2004 - 2009) Impact of the new carbapenem breakpoints. Surg Infect 2012;13:1-7. [http://doi.org/10.1089/sur.2011.074]

8. Bamford C, Bonorchis K, Ryan A, et al. Antimicrobial susceptibility patterns of selected bacteraemic isolates from South African public sector hospitals, 2010. Southern African Journal of Epidemiology and Infection 2011;26:243-250.

9. Kritsotakis EI, Tsioutis C, Roumbelaki M, et al. Antibiotic use and the risk of carbapenem-resistant extended-spectrum- $\beta$-lactamase-producing Klebsiella pneumoniae infection in hospitalized patients: results of a double case-control study I Antimicrob Chemother 2011:66:1383-1391. [http://doi.org/10.1093/jact dkr116]

10. Won SY, Munoz-Price LS, Lolans K, et al. Emergence and rapid regional spread of Klebsiella pneumoniae carbapenemase-producing Enterobacteriaceae. Clin Infect Dis 2011;53:532-540. [http://doi.org/10.1093/ $\mathrm{cid} / \mathrm{cir} 482]$

11. Patel N, Harrington S, Dihmess A, et al. Clinical epidemiology of carbapenem-intermediate or -resistant Enterobacteriaceae. J Antimicrob Chemother 2011;66:1600-1608. [http://doi.org/10.1093/jac/dkr156]

12. Hirsch EB, Tam VH. Detection and treatment options for Klebsiella pneumonia carbapenemases (KPCs) an emerging cause of multidrug-resistant infection. J Antimicrob Chemother 2010;65:1119-1125. [http:// doi.org/10.1093/jac/dkq108]

3. Roberts JA, Kruger P, Paterson DL, et al. Antibiotic resistance - what's dosing got to do with it? Crit Care Med 2008;36:2433-2440

4. Imberti R, Cusato M, Villani P, et al. Steady state pharmacokinetics and BAL concentration of colistin in critically ill patients after IV colistin methanesulfonate administration. Chest 2010;138:1333-1339.

15. Best Care ... Always! www.bestcare.org.za (accessed 17 February 2012). 